CHRONIQUE DE LA RECHERCHE

\title{
SOCIOLOGIE DE LA SCIENCE ET RELATIVISME
}

\section{I. - L'IRRUPTION DE LA SOCIOLOGIE DE LA CONNAISSANCE SCIENTIFIQUE}

En moins d'une quinzaine d'années, le point de vue relativiste a pris une place importante dans les études et les réflexions sur la science, suscitant des travaux théoriques, des recherches empiriques, et aussi des controverses violentes. Il a introduit dans la sociologie des problèmes qui en étaient exclus, réservés à l'épistémologie. Il a changé, ou tenté de changer, l'image dominante de la science, faisant apparaître l'activité scientifique, et donc les affirmations qu'elle produit et qui prétendent être des connaissances valides, comme moins assurées, moins rationnellement fondées, et plus dépendantes du contexte social qu'on ne l'admet habituellement.

Dans les années 30, Mannheim, le fondateur de la sociologie de la connaissance, puis Merton, avaient exclu le contenu de la science du champ de la sociologie de la connaissance, au nom de son objectivité qui rendrait vain tout effort d'explication sociologique. À l'opposé, Spengler qui, à partir de l'exemple des mathématiques, affirmait la relativité culturelle de toute science, était facilement considéré comme un irrationaliste et un obscurantiste. Certains marxistes (Hessen, 1931 ; Borkenau ', 1934) * ont bien tenté de montrer les liens de la science avec l'infrastructure économique, mais leur foi dans la science et donc la possibilité d'une connaissance objective les a toujours empêchés d'aller jusqu'au relativisme. L'effort le plus intéressant pour se sortir de la contradiction entre l'objectivité de la science et sa détermination socio-économique est probablement celui de Lukacs (1924, trad. frçse 1960) pour qui la connaissance est pleinement déterminée par la position de classe, mais pour qui certaines positions dans le système social permettent d'atteindre à une connaissance objective. C'est en particulier le cas des classes ascendantes, la bourgeoisie au XVIII ${ }^{e}$ siècle, la classe ouvrière lorsqu'il écrivait au début du XX⿳亠口冋. Luttant pour

* Les noms d'auteurs suivis d'une date renvoient à la Bibliographie p. 288-290.

1. Jusqu'à très récemment, Borkenau n'était guère connu, au moins en France, que par quelques lignes destructrices de Koyré (1943, repris dans KoYRÉ, 1973, p. 167). Un numéro des Cahiers S.T.S. ( $\left.\mathrm{N}^{\circ} 7,1985\right)$ présente quelques textes de lui et des commentaires permettant de se faire une meilleure opinion.

Revue de synthèse : IV ${ }^{e} \mathrm{~S} . \mathrm{N}^{\circ} 3$, juillet-septembre 1986. 
leur émancipation, allant dans le sens de l'histoire, elles n'ont pas besoin de déformer la réalité et peuvent donc atteindre à une véritable connaissance.

L'idée qu'il existe ainsi des points de vue privilégiés a été reprise par d'autres pour éviter le relativisme ou le scepticisme tout en acceptant la détermination sociale des connaissances ; ce fut le cas de Mannheim, pour qui cette position était celle des intellectuels indépendants, et plus récemment celle de Lukes (1973) qui en fait la base d'une critique de principe du relativisme, sans préciser d'ailleurs quelle serait cette position privilégiée.

À la suite de Merton, la sociologie de la science s'est séparée de la sociologie de la connaissance, et s'est orientée vers l'étude du fonctionnement de la communauté scientifique et de ses institutions. C'est ainsi qu'ont été étudiées les normes de la science (Merton, 1942), son système de récompenses (Hagstrom, 1965 ; Storer, 1966) son développement quantitatif (Price, 1963), les hiérarchies et le prestige (Cole, Cole, 1973), les facteurs de productivité (Pelz, Andrews, 1966), les relations avec la politique (Salomon, 1970), les conditions institutionnelles d'émergence de nouvelles disciplines (Ben David, Collins, 1966), la division du travail dans les laboratoires (Lemaine, Darmon, El Nemer, 1983), les motivations des chercheurs (Maslow, 1969), etc. ${ }^{2}$.

Mais le contenu même des connaissances scientifiques échappait, et devait échapper, à l'analyse et à l'explication sociologiques. Tout au plus admettait-on qu'on puisse expliquer par des facteurs sociaux ou psychologiques des erreurs ou des retards, des orientations particulières de la recherche en un lieu et à un moment donné, plus généralement tous les accidents d'un développement dont le mouvement général se déduit seulement de sa dynamique interne ${ }^{3}$. Bachelard (1934) a analysé les " obstacles épistémologiques " qu'il a fallu surmonter pour que se constitue une physique scientifique, et plus largement la science moderne. Mais une fois ces obstacles levés ou contournés, c'est la logique interne du développement de chaque science qui s'impose. Selon ces perspectives, qui convergent au moins sur ce point, il ne saurait y avoir de véritable psychologie ou sociologie de la connaissance scientifique, si ce n'est pour éclairer des aspects mineurs ou accidentels de son histoire 4 .

Depuis la fin des années 60 , les points de vue ont été profondément transformés. Une nouvelle génération de sociologues a sauté par-dessus les barrières dressées par les grands ancêtres, affirmant que la science est soumise

2. On trouvera un bon exposé de ces travaux, et de l'évolution vers la sociologie de la science actuelle dans LECUYER (1978).

3. Un exemple particulièrement net de cette position est fourni par LAKATOS (1971) qui propose de procéder à une " reconstruction rationnelle de l'histoire des sciences " qui la débarrasserait de ces accidents et pourrait alors servir de matière première à des études épistémologiques.

4. Rappelons à ce propos un argument célèbre, dont il n'est pas facile de retracer l'origine : si Beethoven était mort à 10 ans, nous n'aurions rien de son cuvre, alors que si Newton était mort au même âge, il est certain que nous aurions la même physique, qui serait tout au plus apparue un peu plus tard. Que cette conjecture soit presque toujours jugée évidente montre bien la conviction quasi générale que la science a un développement plus nécessaire, une logique plus contraignante que les autres phénomènes dont on peut faire l'histoire. 
aux mêmes déterminismes que les autres activités humaines, qu'elle n'a pas de position privilégiée, et donc qu'il n'y a pas de raison pour qu'elle échappe à l'investigation scientifique.

Un indice frappant de ce changement d'attitude se trouve dans le contenu de deux recueils d'articles parus en Grande-Bretagne à une dizaine d'années d'intervalle. Dans le premier, Rationality (Wilson, 1970), on se demande si, et dans quel sens, on peut considérer que les croyances acceptées dans d'autres cultures peuvent être considérées comme rationnelles. On y discute sur la rationalité des oracles des Azandé ou des Cultes du Cargo mélanésiens. En 1982, paraît un second recueil, Rationality and Relativism (Hollis, Lukes, 1982), avec des références explicites au premier, et quelques auteurs communs. Cette fois, autant que les cultures exotiques, c'est la science qui est en question, la science considérée comme un « système organisé de croyances » mis sur le même plan que les autres. Les convictions des physiciens nucléaires posent aux sociologues et aux épistémologistes les mêmes problèmes que celles des Azandé. Même si, en fin de compte, la majorité des auteurs affirment des positions antirelativistes, au moins en ce qui concerne la science, tous reconnaissent la réalité du problème et éprouvent le besoin de prendre position par rapport à lui. En onze ans, le contexte intellectuel a bien changé. Le relativisme des anthropologues, forme de respect des cultures différentes et de reconnaissance de leurs spécificités, s'est installé maintenant au cœur même de notre civilisation, dans ce qu'elle a de plus spécifique $^{5}$. Elle est perçue comme un ensemble de systèmes de croyances hétérogènes, qu'il faut mettre sur le même plan que les croyances exotiques, et qu'il est inutile de chercher à comparer, faute de critères absolus qui permettraient d'affirmer la validité, ou simplement la supériorité, de l'un d'entre eux.

Sous l'influence de cette nouvelle génération de sociologues, des problèmes qui restaient du ressort de l'épistémologie, ou d'une histoire des sciences très philosophique comme celle de Koyré ou de Bachelard, sont apparus comme pouvant relever d'investigations sociologiques empiriques : on a discuté de la continuité ou de la discontinuité du développement scientifique, de l'importance relative des facteurs internes ou externes, de l'universalité ou de la localité de la rationalité, etc.

\section{II. - PREUVE ET CONSENSUS}

Avant d'exposer et de discuter en détail les idées relativistes (le pluriel est nécessaire car, en fait, on peut en distinguer plusieurs, que les nécessités de la polémique amènent à confondre de part et d'autre), nous donnerons une première idée des positions en présence sur un exemple, la manière de traiter

5. Cela se produit, curieusement, au moment où les anthropologues ont recommencé à chercher des invariants trans-culturels, à la suite de Lévi-Strauss, Mary Douglas et d'autres. 
les relations entre preuve et consensus, deux termes fréquemment discutés dans ce contexte.

Ce sont deux notions apparemment très éloignées, l'une logique, l'autre sociale. Et pourtant, paradoxalement, les positions qui s'affrontent aujourd'hui sur la nature de la connaissance scientifique les confondent, ou du moins voient en elles des liens si étroits qu'il devient impossible de les distinguer, mais ce rapprochement se fait sur des arguments tout à fait opposés.

Pour ceux qu'on appellera, par commodité, les rationalistes (Laudan, 1977 ; Lakatos, 1978 ; Hollis, 1982) une preuve correcte s'impose par elle-même, au moins aux personnes compétentes et sans préjugés, et donc elle doit normalement entraîner le consensus. Si celui-ci n'est pas atteint, c'est faute d'informations suffisantes, ou suffisamment décisives, parce que des préjugés idéologiques aveuglent, parce qu'on reste trop attaché à des points de vue antérieurs, etc. Le consensus s'explique par la valeur empirico-logique de la preuve, le non-consensus par des facteurs extérieurs, psychologiques ou sociologiques. Les controverses scientifiques s'expliquent soit par de tels obstacles externes, soit par la difficulté d'administrer des preuves décisives.

À l'opposé, le relativisme, au moins sous sa forme sociologique actuelle, se refuse à reconnaître $a$ priori des critères absolus, universels, de rationalité. Plus exactement, il se refuse à se placer en position de savoir ce qu'ils sont, et il se borne à constater, du moins il le prétend, que ce qu'on accepte comme argument, ou ce qu'on qualifie de rationnel, n'est pas identique dans différents contextes. Ce qui est reconnu comme preuve ne l'est que de façon locale, à l'intérieur d'un système de croyances. Le sociologue n'a pas à se prononcer sur ce qui est rationnel et ce qui ne l'est pas, mais il peut constater que, à un certain moment, un argument a été accepté comme preuve par un certain groupe, et qu'il a entraîné le consensus. Celui-ci n'est donc plus la conséquence d'une nécessité logique qui s'imposerait à tous, mais l'indice que l'argument a été reconnu par les intéressés comme conforme aux critères de preuve du groupe. Ces critères étant locaux, dépendants du contexte, ils ne peuvent pas être considérés comme dérivant d'une logique universelle, mais ils relèvent d'une explication sociologique, au même titre que n'importe quelle autre croyance ou norme. Dans cette perspective, le consensus est purement social, résultat d'interactions, de négociations entre chercheurs ayant des ressources et des pouvoirs différents, des " intérêts cognitifs " différents ou parfois convergents, mais qui partagent un même système de croyances, celui de leur communauté. Ces croyances partagées, qui permettent le consensus, résultent elles-mêmes de négociations et de consensus antérieurs dont l'origine sociale a été occultée, et qui donc apparaissent comme « vraies », " objectives » ou « naturelles » à ceux qui les acceptent. Mais ces termes représentent des catégories utilisées par les acteurs, et non une réalité qui les transcenderait. En eux-mêmes, ils n'expliquent rien, c'est leur usage qui doit être expliqué par le sociologue.

Ce bref exemple met bien en évidence le cœur du relativisme : la conviction que tout est social, qu'il n'y a pas d'universel, et pas de point de vue absolu, 
privilégié, qui justifierait les prétentions de la connaissance scientifique à une quelconque supériorité 6 .

\section{III. - LE NOYAU DUR DU RELATIVISME}

Le relativisme n'est pas une théorie. Certes, les auteurs qui s'y rattachent ont publié de nombreux textes théoriques, mais ils y présentent plutôt une théorie de la production des connaissances scientifiques, dont le relativisme apparaît comme une conséquence. Néanmoins, c'est le plus souvent celui-ci qui est mis en évidence.

Ce n'est donc pas une tentative de représentation de la réalité, mais plutôt un ensemble de points de vue, de règles, de méthodes, de convictions. Et aussi, peut-être, au départ, surtout des refus : refus de la prééminence actuellement reconnue à la science par rapport à d'autres formes de connaissance, refus de croire à une rationalité universelle et intemporelle, refus d'épistémologies qui ignorent les aspects sociaux des pratiques de recherche.

Collins (1981a), plutôt que de théorie, parle du « programme de recherches » (" research program ") relativiste, dans le sens qu'a donné Lakatos (1978) à cette expression. C'est effectivement une source d'orientations pour des recherches empiriques : à côté de leurs textes méthodologiques ou théoriques, les relativistes ont produit un grand nombre d'études empiriques en histoire et en sociologie des sciences, et c'est souvent à propos de ces recherches que leurs idées plus générales s'expriment ?

Comme tout courant de pensée, le relativisme constitue un ensemble complexe. Certains auteurs revendiquent le terme pour eux-mêmes (Barnes, Bloor, Mc Kenzie, Collins, Pickering, Pinch, etc.). D'autres non, mais soutiennent des points de vue très proches, ou admettent implicitement certains aspects du relativisme dans leurs recherches (Latour, Callon, Knorr-Cetina, Forman, Feyerabend ${ }^{8}$,

6. Selon ce point de vue, la distinction, très discutée par de nombreux épistémologistes, surtout anglo-saxons, entre « connaissance » et " croyance » n'a plus de raison d'être. Si on admet, comme on le fait d'habitude, qu'une connaissance est une " croyance justifiée ", alors il faut accepter un critère extérieur de justification. Or, c'est ce que les relativistes refusent. Normalement, ils devraient admettre qu'ils font la sociologie de la croyance, et qu'une sociologie de la connaissance n'a pas de sens.

7. La Bibliographie, en fin d'article, donne les références qui nous ont semblé les plus importantes ou les plus caractéristiques. On trouvera la traduction française d'articles parmi les plus représentatifs dans deux volumes publiés par PANDORE (1982 et 1985).

8. Le cas de Feyerabend est très particulier, et il est peut-être abusif de le ranger parmi les relativistes. En fait, son célèbre principe "Tout est bon " ("Anything goes ") (FEYERABEND, 1975) peut être considéré comme l'affirmation d'un relativisme quant à la méthode. Néanmoins, il semble croire à la validité de la connaissance scientifique, même si, comme certains relativistes, il refuse la prééminence sociale de la science et, par exemple, soutient les demandes des «créationnistes " américains, qui réclament l'enseignement de la conception biblique de la création en parallèle à celui de la théorie de l'évolution, ou la légitimité des recherches en parapsychologie ou en astrologie (Feyerabend, 1978). Mais son point de vue est plus politique : il préconise, selon son expression, la « séparation de la science et de l'État ». 
Brannigan, etc.). Parmi ceux qui se reconnaissent relativistes, l'accord n'est d'ailleurs pas total, bien qu'ils ne polémiquent guère entre eux ; les différences d'accent mis sur tel ou tel argument sont notables.

Le " noyau dur " du relativisme, pour reprendre encore une expression de Lakatos, peut se résumer en quelques citations : « no particular set of natural beliefs can be identified as reasonable, or as uniquely the truth " (Barnes, 1974, p. 22). " The natural world has a small or non-existent role in the construction of scientific knowledge " (Collins, 1981 a).

Si on ne rend pas compte des croyances scientifiques par une réalité extérieure, par une nature, alors qu'est-ce qui peut les expliquer ? Pour tous les relativistes, la réponse est claire, du moins dans une première formulation : ce qui est premier, ce sont les pratiques des chercheurs qui « construisent " les phénomènes qu'on considérera ensuite comme une réalité objective. Et ces pratiques se déroulent dans un contexte qui les oriente ou les détermine, qu'il s'agisse de la société globale ou de la communauté particulière à laquelle appartiennent les chercheurs.

Les relativistes, il est intéressant de le remarquer, sont professionnellement des sociologues ou des historiens très sociologisants, et non des philosophes ${ }^{9}$. Et ces sociologues sont en réaction à la fois contre l'absence quasi totale du social dans les descriptions ou les justifications que les épistémologistes donnent du développement des connaissances scientifiques, contre la sociologie classique de la science, qui étudie le fonctionnement de la communauté scientifique et de ses institutions, mais s'interdit de s'occuper du contenu même de la science, et contre une histoire des sciences qui n'est qu'une histoire d'idées désincarnées.

Autour de ces convictions communes, les arguments diffèrent, incitant à des recherches empiriques très variées. Nous en avons distingué quelques grandes catégories, qui nous guideront pour examiner et discuter cet ensemble complexe.

\section{IV. - SPÉCIFICITÉ OU BANALITÉ DE LA SCIENCE}

Un premier groupe d'arguments a été exprimé particulièrement par Barnes $(1974,1977,1982)$. Ils se rattachent à la conception du développement scientifique proposée par Kuhn $\left(1962,2^{e}\right.$ éd. 1970), et plus précisément à la notion de paradigme qu'il a introduite, et à ce qu'il appelle l'incommensurabilité des paradigmes ${ }^{10}$. Ces conceptions sont maintenant suffisamment connues pour qu'on puisse se borner à rappeler rapidement ce qui est directement important pour notre discussion. Selon Kuhn, un paradigme est un ensemble de théories, de concepts, de données empiriques reconnues, de méthodes, acceptées par

9. La seule exception notable est D. Bloor, mais il fait partie du « Science Study Group » de l'Université d'Edimbourg qui, sauf erreur, ne comprend, à part lui, que des sociologues ou des historiens.

10. Les conceptions de Kuhn sont sans aucun doute à l'origine de certaines formes de relativisme, mais on ne saurait pour autant affirmer qu'il les accepte. Sur ce point, son attitude est ambiguë, mais il est au moins clair que les conséquences extrêmes que certains tirent de ses idées ne lui plaisent guère. 
une communauté scientifique. C'est une conception du monde, une manière de percevoir et d'organiser la réalité. Ce qui est important pour notre propos, et qui a été explicitement repris par Barnes, c'est l'idée qu'un paradigme est fermé sur lui-même, et qu'il comprend ses propres critères de validité. On ne peut donc pas critiquer un paradigme du point de vue d'un autre ; le choix entre deux paradigmes concurrents ne peut donc pas être purement rationnel "1, bien qu'on ne voie pas très clairement ce que peuvent être, dans cette perspective, les critères de choix.

Kuhn s'intéresse exclusivement aux paradigmes scientifiques. Barnes élargit la problématique : il estime qu'il y a chez Kuhn une sociologie générale qu'il n'a pas explicitée. La notion d'incommensurabilité est étendue par lui à tous les systèmes de croyances, la science n'étant, dans cette perspective, qu'un système parmi d'autres, qui sont, par exemple, la magie ou la religion. D'une certaine façon, cet élargissement, qui n'intéresse guère Kuhn dont les analyses restent toujours internes à la science, peut renforcer son point de vue : on peut, en effet, discuter largement de l'incommensurabilité des paradigmes scientifiques concurrents et soutenir qu'ils sont en fait, par certains aspects, comparables. Mais ce point de vue est plus fort lorsqu'il s'agit, par exemple, de comparer la science et une religion. Les « preuves " empiriques, essentielles dans le système de croyance de la science, peuvent n'avoir aucune pertinence, aucune valeur de conviction pour un croyant. Inversement, la foi dans un texte révélé ou une expérience mystique n'existe tout simplement pas dans le paradigme scientifique ${ }^{12}$.

L'incommensurabilité des paradigmes se manifeste parfois par le sentiment d'incongruité que l'on éprouve devant des tentatives d'utiliser des éléments d'un certain paradigme à l'intérieur d'un autre. Citons comme exemple un médecin positiviste du milieu du XIX $^{c}$ siècle qui, paraît-il, se serait procuré une hostie consacrée, l'aurait analysée et proclamé triomphalement n'y avoir trouvé aucune trace de chair... Mais les frontières ne sont pas toujours aussi étanches. Sur le même thème des relations entre paradigmes scientifiques et religieux, on peut citer des exemples où la transposition est beaucoup plus acceptable. Ainsi, la controverse qui s'est déroulée en Grande-Bretagne à partir de 1872 sur l'efficacité des prières pour les malades constitue un cas limite : certains croyants ont accepté l'idée d'une vérification expérimentale, d'autres l'ont jugée tout à fait scandaleuse. Manifestement, pour ces derniers, les paradigmes étaient incommensurables, pas pour les premiers. (Cette discussion est racontée par Brush, 1978). On peut encore citer un troisième exemple, cette fois de compatibilité acceptée : la commission chargée de contrôler les miracles de Lourdes veut appliquer des critères strictement conformes à la méthodologie scientifique, tout en croyant que des guérisons miraculeuses sont possibles. On voit que, si les

11. C'est du moins l'interprétation qu'en donne Barnes (1982, chap. 4). Pour Kuhn lui-même, un paradigme en remplace un autre lorsque le premier comprend trop d'anomalies, et que le second permet d'en rendre mieux compte. Il y a donc bien une comparaison possible, même si d'autres critères peuvent intervenir.

12. Cela n'empêche pas un même individu de pouvoir partager les deux systèmes de croyances, bien au contraire. C'est précisément parce qu'ils sont incommensurables que certains scientifiques peuvent être en même temps croyants. 
paradigmes religieux et scientifiques sont bien distincts, la coupure entre eux n'est pas uniformément étanche et, sur certains points, ils peuvent ne pas être incommensurables.

Dans la rhétorique relativiste, les deux niveaux d'incommensurabilité, entre paradigmes scientifiques et entre la science et d'autres systèmes de croyances, jouent chacun leur rôle. Celle entre paradigmes scientifiques permet de mettre en cause la notion de vérité, et l'idée de progrès scientifique considéré comme un progrès objectif. L'incommensurabilité entre grands systèmes de croyance (entre formes de vie, disent Barnes ou Bloor, reprenant une expression de Wittgenstein) leur sert à refuser toute position privilégiée à la connaissance scientifique.

La valeur de ces arguments dépend évidemment de la croyance que l'on peut avoir dans l'incommensurabilité des paradigmes. Nous venons de voir que des systèmes de croyances peuvent ne pas être aussi hétérogènes qu'on l'affirme parfois. À l'intérieur de la science, ce problème est une des formes que prend, actuellement, la controverse qui oppose périodiquement, en histoire des sciences, les partisans d'une représentation continue du développement scientifique à ceux d'une conception discontinue. Mais peut-être, posé ainsi sous la forme d'une opposition stricte, n'est-ce qu'un faux problème : tout système de croyances, scientifique ou autre, est un ensemble complexe dont les éléments sont nombreux et divers. On peut soutenir que certains évoluent de façon continue ou restent invariants au moment même où d'autres subissent une rupture. Surtout depuis que les différentes disciplines scientifiques sont bien établies (sont passées du stade pré-paradigmatique au stade paradigmatique, pour parler comme Kuhn, ou ont réalisé leur coupure épistémologique, si l'on utilise le langage de Bachelard et de ses successeurs), chaque "révolution " a intégré les acquis antérieurs et a maintenu certains principes, concepts ou méthodes. $\grave{A}$ quoi les discontinuistes, Kuhn en particulier, rétorqueraient que ce qui caractérise une révolution scientifique, ce n'est pas la modification de tel ou tel point particulier, mais une transformation globale du paradigme, qui fait que, même si un élément semble identique à son correspondant dans le paradigme précédent, les relations qu'il entretient avec le reste du système sont changées. Néanmoins, le fait même que de telles correspondances soient possibles (ce qui n'est guère le cas lors de la " coupure épistémologique ») introduit un élément de continuité.

Qu'on pense, par exemple, à la masse dans la mécanique classique ou dans la mécanique relativiste; il y a entre elles des différences essentielles, la plus évidente étant que l'une est constante alors que l'autre est fonction de sa vitesse. Mais elles jouent des rôles très semblables, par exemple dans la relation entre la force et l'accélération, l'expression de l'énergie cinétique, ou dans la loi de la gravitation. Mais, autre différence essentielle, cette loi a une place complètement différente dans les deux systèmes : postulée chez Newton, elle se déduit de postulats plus généraux dans la théorie de la relativité générale. Continuité ou discontinuité ? Les deux, peut-être, ce qui rend difficile de soutenir que les paradigmes sont complètement fermés sur eux-mêmes. 
Le problème se pose de la même façon, avec les mêmes ambiguités, lorsqu'on compare des systèmes de croyances différents, en particulier des croyances scientifiques à d'autres. Si la part de continuité et de discontinuité peut varier d'un cas à l'autre, il n'en reste pas moins que des éléments communs se retrouvent toujours, comme aussi des éléments spécifiques. D'ailleurs, une forme de relativisme consiste précisément à abaisser les barrières entre les différentes formes de pensée, et à soutenir que la pensée scientifique est imprégnée de la vie quotidienne et de croyances de sens commun, ce qui devient un argument contre sa rationalité spécifique.

En effet, paradoxalement, les relativistes, qui pourtant insistent sur la spécificité du système de croyances scientifiques, banalisent en même temps la science. Sous-jacents à la plupart de leurs écrits, on trouve : "Voyez, la science, ce n'est pas si extraordinaire, ce n'est pas tellement différent des pratiques et des croyances quotidiennes, ou de celles qui règlent d'autres activités. " Latour et Woolgar (1979, p. 153), analysant des critères proposés pour repérer les spécificités de la démarche scientifique, s'efforcent de démontrer qu'on les retrouve dans la vie quotidienne. Plus tard, Latour (1983) parle de « redistribuer le grand partage " (entre la science et le sens commun, entre la pensée civilisée et la pensée sauvage, etc.) ; en fait, il s'agit bien de l'annuler. Knorr-Cetina (1981) insiste sur l'" opportunisme " des chercheurs, plus guidés par le désir d'être reconnus que par le souci de la vérité. De nombreux autres auteurs s'attachent à montrer que l'activité scientifique est plus approximative, moins rigoureuse, moins « propre " que ne le montrent les publications et les manuels ${ }^{13}$. On ne peut donc pas caractériser la science par sa rigueur. Barnes (1974), lorsqu'il veut préciser ce qui fait la spécificité des sciences de la nature, ne trouve à mentionner que quelques traits qui, nous avons pu le constater, laissent ses lecteurs insatisfaits. Il énumère :

" a priori opposition to theological arguments and theorizing, aversion to anthropomorphic or animistic theoretical entities, an insistant differentiation between fact and value, a cosmology firmly denying man any special significance, and, finally, a tendency to reject or devalue scientific work on hypnotism, extra sensory perception or similar topics » $(1974$, p. 45$)$.

Plus loin, il parle du rejet de modes de pensée ou d'expression " idiosyncrasiques".

Ce dernier point, sur lequel il n'insiste d'ailleurs pas beaucoup, est peut-être le plus important : il exprime le caractère " public » de l'activité scientifique et de ses résultats. Mais on ne peut s'empêcher de penser qu'il y a autre chose. À force de ne vouloir s'intéresser qu'aux aspects sociaux de la science, de

13. Ceux-ci sont la bête noire des relativistes, au moins en tant que documents sur ce qu'est réellement la science. $\dot{A}$ leurs yeux, ils donnent une image tout à fait irréaliste de l'activité scientifique. Par contre, pour Kuhn, dont les intérêts sont, il est vrai, différents, la lecture des manuels, qui contiennent ce qu'une communauté scientifique cherche à transmettre à ses futurs membres, constitue la meilleure voie d'accès à la connaissance d'un paradigme. 
s'efforcer d'en parler comme de toute autre activité, les relativistes perdent de vue sa spécificité. En lisant leurs travaux, on apprend beaucoup sur la vie des chercheurs, on s'aperçoit qu'ils sont des hommes comme les autres, aussi sujets à des erreurs ou à des influences, que ce ne sont pas de pures machines logiques. Mais finalement, on sait peu de choses sur ce qui fait qu'ils sont chercheurs, et pas prêtres, commerçants ou ferblantiers... ${ }^{14}$.

\section{V. - LE PRINCIPE DE SYMÉTRIE}

La deuxième idée directrice du relativisme apparaît comme une règle de méthode, mais elle est bien plus que cela : elle exprime, telle qu'elle est appliquée, des présupposés à l'égard du social et de la logique (ou, plus largement, du cognitif). Elle a été exprimée pour la première fois par D. Bloor (1976), qui en a fait l'élément central du « programme fort " qu'il a proposé à la sociologie de la science. C'est le principe de symétrie dans l'explication : en essayant de rendre compte des croyances scientifiques, il faut traiter de la même manière, expliquer par les mêmes causes, les croyances vraies et les croyances fausses, celles qui sont acceptées et celles qui sont rejetées.

Ce principe a l'air évident, mais on en appréciera mieux la portée en la situant par rapport à ce à quoi il s'oppose, la thèse rationaliste, pour laquelle les croyances vraies, les connaissances, ne s'expliquent que par leur vérité. Nous avons déjà rencontré cette position à propos des relations entre preuve et consensus. Elle a été exprimée plusieurs fois, et récemment avec une concision et une clarté particulières par Hollis (1982) : "True and rational beliefs need one sort of explanation, false and irrational beliefs another. "

Ce principe soulève des problèmes particulièrement graves en sociologie de la science, mais il peut s'appliquer à de nombreux autres domaines. De nombreuses études criminologiques, par exemple, ne se posent aucune question sur les comportements non délinquants ou non déviants, comme si le respect des lois et des normes était normal et ne demandait aucune explication, seul l'écart posant problème.

On peut donner de ce principe deux interprétations très différentes dans leur portée. Selon la première, ce serait une pure règle de méthode, qui ne préjugerait en rien des conclusions. Il s'agirait, au départ, de ne privilégier aucun mode d'explication et, surtout, d'admettre que rien ne va de soi. Ce serait donc une nécessité pour le sociologue qui veut, qui doit, prendre une distance suffisante par rapport aux a priori de la société dans laquelle il vit et travaille. Cette conception serait, en principe, compatible avec des conclusions rationalistes : on pourrait montrer empiriquement, par exemple, que des croyances vraies

14. FEYERABEND (1970) faisait remarquer à Kuhn que sa description de l'activité scientifique n'avait rien de spécifique, et il s'amusait à décrire, avec les concepts kuhniens, l'activité d'une bande de gangsters... 
sont acceptées parce qu'elles sont fondées sur des expériences et des déductions solides, alors que les erreurs, ou les refus d'accepter la vérité, s'expliqueraient par des facteurs sociaux qui perturberaient certains chercheurs. La symétrie serait au départ, mais pourrait ne pas se retrouver à l'arrivée. L'autre interprétation est plus proprement relativiste, et c'est probablement celle qui a la préférence des auteurs qui préconisent ou adoptent le principe de symétrie : ce serait la conviction, ou le postulat, que le même type d'explication s'applique en fait à toutes les croyances, indépendamment de leur validité. Mais, chez eux, la symétrie ne s'accompagne pas de la prise en considération de toutes les explications possibles : sans toujours le dire explicitement, ils ne se posent aucune question sur les facteurs cognitifs qui pourraient éventuellement intervenir, se contentant de montrer que des facteurs sociaux interviennent toujours, oubliant que ce n'est pas la même chose que de montrer que les facteurs cognitifs n'interviennent jamais.

En tant que règle de méthode, ce principe n'est guère attaquable, encore qu'il ne soit pas facile à appliquer à tout coup, en sociologie de la science ou dans d'autres domaines ${ }^{15}$. Surtout, il s'impose si le chercheur ne veut pas prendre parti, ne veut pas prétendre savoir où sont la vérité et l'erreur. La formule de Hollis citée ci-dessus est claire, et elle est argumentée dans la suite de son texte, mais on reste en droit de lui demander comment il sait ce qui est vrai et rationnel, et comment il le distingue du faux et de l'irrationnel. S'il prétend disposer de critères pour cela, il suppose le problème résolu, et la seule question qui subsiste est effectivement d'expliquer pourquoi tout le monde n'a pas la même lucidité que lui. Si on s'y refuse, si, par principe, on se veut agnostique, on en est réduit au seul critère sociologique, celui de la constatation de l'acceptation ou du rejet par la communauté concernée.

Mais ce critère, lui aussi, exige une rupture de la symétrie : il faut expliquer l'acceptation ou le rejet, et pour cela il faut bien accepter que les options en présence diffèrent. Le principe de symétrie doit nécessairement aboutir à une dissymétrie. Il s'agit de savoir laquelle. Ici encore, on peut retrouver la position rationaliste, et conclure que la dissymétrie porte sur les aspects logiques et cognitifs qui seraient décisifs dans l'acceptation d'une croyance vraie et non dans celle d'une erreur. Toutefois, les relativistes ont une préférence manifeste pour des différences plus faibles, qui ne porteraient que sur l'importance relative de certains facteurs.

L'exemple le plus souvent cité d'application du principe de symétrie à un cas concret est un article de Farley et Geison (1974) sur la controverse entre Pasteur et Pouchet à propos de la génération spontanée, article d'ailleurs antérieur à la formulation du " programme fort » par Bloor. Les idéologies des deux protagonistes y sont analysées, et les auteurs montrent que Pasteur a été tout autant guidé par son idéologie que son adversaire vaincu. Mais, sur le même thème, Latour (1984) pousse l'analyse plus loin : il ne se contente pas de se demander pourquoi Pasteur a cru aux microbes, mais pourquoi il a triomphé. C'est le

15. Comment expliquer de façon symétrique, par exemple, l'état de santé ou une infection? 
succès qui intéresse Latour, et à ce moment il est clair que l'explication ne peut être que dissymétrique.

Néanmoins, pour Latour, la symétrie est ailleurs : elle consiste avant tout dans le refus de la solution dissymétrique banale : Pasteur a triomphé parce qu'il avait raison, parce que ses expériences étaient plus convaincantes et ses explications plus rationnelles. Il essaye, au contraire, de montrer tous les facteurs extrascientifiques, sociopolitiques, qui ont entraîné la victoire du pasteurisme, et qui imprégnaient tout autant ses adversaires.

Les relativistes voient dans le principe de symétrie plus qu'une règle de méthode. Ils sont convaincus que le même type d'explication rend compte de toutes les croyances. C'est ce que Barnes et Bloor (1982) appellent le monisme, qu'ils présentent comme le refus de séparer le social et le cognitif, les facteurs internes et externes. Mais, en fait, on sent bien leur penchant pour les explications purement sociologiques, donc externes.

\section{VI. - CONTEXTE MACRO-SOCIAL, CONTEXTE MICRO-SOCIAL}

Cela nous amène à la troisième composante du relativisme, peut-être la plus importante, la conviction (qu'on cherche à étayer par des études empiriques) que les croyances scientifiques s'expliquent totalement ou principalement par des déterminants sociaux, et non par la logique interne, autonome, du développement de la recherche.

Cette insistance sur les facteurs sociaux ne résulte pas seulement de constatations empiriques. Elle est une conséquence nécessaire du refus de prendre en considération des critères absolus de validité ou de rationalité, et de la négation même de leur possibilité. Parfois, plus modestement, ils reprennent la thèse de Duhem sur l'indétermination de la théorie par les faits (par exemple, Pickering, 1984 a). Si on accepte cette position, il faut bien admettre que d'autres facteurs interviennent pour tirer d'une expérience une conclusion assurée. Le recours aux déterminants sociaux est une manière de résoudre ce problème, d'expliquer comment on est sorti de l'indétermination.

Toutefois, les différents auteurs ne donnent pas le même contenu au « social ". Pour les uns (Barnes, Mc Kenzie, Forman, Latour), c'est la société globale, ou tout au moins des aspects de celle-ci qui restent externes à la communauté scientifique. Pour d'autres (Collins, Pinch, Pickering, Latour dans son premier ouvrage), c'est ce qui se passe à l'intérieur de celle-ci qui est déterminant. On se trouve donc devant deux types d'explications sociologiques, dont les implications sont très différentes.

Le premier groupe, que l'on peut qualifier sommairement de macrosociologique, s'intéresse donc aux relations entre l'activité scientifique et la société globale, dont ces auteurs rappellent fortement que les chercheurs sont aussi membres. Contrairement à l'autre courant, qui procède par des analyses fines du comportement des chercheurs et de leurs interactions, les auteurs en question font le plus souvent des analyses historiques (même si celles-ci 
vont jusqu'au présent), avec l'accent mis sur le contexte socio-politique ou économique.

Nous en examinerons un seul exemple, très souvent cité et particulièrement caractéristique, celui de Forman (1971) qui étudie les développements de la théorie quantique dans les années 20 et plus précisément l'abandon de la conception classique de la causalité, qu'il explique par l'idéologie dominanté, antiscientiste, voire antiscientifique, de l'Allemagne de Weimar. La thèse de Forman est que l'abandon de la causalité était une manière, pour les physiciens, de s'adapter à un environnement hostile, et de retrouver leur audience perdue. Pour cela, il ne s'appuie pas sur des textes scientifiques mais sur des documents non scientifiques produits par des physiciens : discours, articles, déclarations publiques, et il montre que ceux qui, dans des contextes non scientifiques, acceptaient le plus facilement les critiques adressées à la science et à sa représentation mécaniste du monde, ont été aussi ceux qui, les premiers, ont introduit ou accepté l'indéterminisme de la mécanique quantique.

On peut considérer cette publication comme un bel exemple de démonstration de l'influence de facteurs externes, bien qu'on puisse objecter que, ayant délibérément exclu de son analyse les textes proprement scientifiques, Forman n'avait aucune chance de pouvoir montrer le rôle des facteurs internes et de la dynamique proprement scientifique ${ }^{16}$.

Cette étude est importante par son thème, son ambition, la richesse de sa documentation et la minutie de l'analyse. Néanmoins, indépendamment des critiques techniques qu'on a pu lui adresser, on peut se demander ce que Forman a vraiment démontré. Si on prend au sérieux son affirmation centrale, l'existence d'un lien causal entre l'idéologie de Weimar et l'indéterminisme quantique, cela voudrait dire que ci cette configuration idéologique particulière ne s'était pas produite, la mécanique quantique ne serait pas apparue, ou aurait pris une autre forme que l'indéterminisme. Une affirmation aussi radicale est peu vraisemblable. Par contre, on peut supposer qu'un contexte critique à l'égard de la causalité a pu contribuer à lever un " obstacle épistémologique " et donc à accélérer l'apparition ou l'acceptation de l'indéterminisme. Ou encore, on estimera que Forman explique pourquoi la mécanique quantique est apparue en Allemagne et non dans un des autres pays scientifiquement aussi avancés. Mais, évidemment, ces remarques qui réduisent considérablement la portée de l'article, se placent du point de vue de la " science sanctionnée ", celle qui est admise aujourd'hui, et n'envisage pas qu'une physique différente ait pu apparaître. Or, c'est bien ce qu'admet implicitement le relativisme, même si, à notre connaissance, aucun de ceux qui s'en réclament n'a jamais explicitement soutenu une thèse aussi extrême. Nous reviendrons sur ce point dans la conclusion.

16. C'est d'ailleurs une critique générale, qu'on peut adresser à la plupart de ceux qui tiennent à un type d'explication, qu'il soit internaliste ou externaliste : ayant montré qu'une catégorie de facteurs intervient, ils en concluent sans plus que l'autre ne joue aucun rôle. Mais, dans le cas de l'indéterminisme quantique, HENDRY (1980) a pu faire une analyse purement interne, montrant les problèmes proprement scientifiques que l'abandon de la causalité permettait de résoudre. Ce qui est curieux, c'est que chacun estime avoir réfuté l'autre. 
Le deuxième courant réalise des études détaillées des pratiques des chercheurs, soit par des entretiens (Collins, 1975, 1981 b ; Travis, 1981 ; Pinch, 1981 ; etc.), soit par observation directe, en participant à la vie d'un laboratoire, établissant avec lui la même relation que l'anthropologue avec les cultures qu'il étudie (Latour, Woolgar, 1979 ; Knorr-Cetina, 1981). Ce qu'ils trouvent est très loin de l'image traditionnelle du savant qui utilise des méthodes éprouvées et rigoureuses, que présentent la plupart des publications scientifiques. La réalité qu'ils décrivent est beaucoup plus incertaine, problématique. Collins (1975), dans une des premières recherches effectuées dans cette perspective, étudie la réplication d'une expérience, et constate que cette notion, méthodologiquement fondamentale, est loin d'être claire. L'acceptation d'une expérience comme étant la répétition et donc la vérification d'une autre doit être négociée. Ce terme, déjà largement utilisé par l'interactionnisme symbolique et l'ethno-méthodologie, va devenir central dans cette description des pratiques scientifiques. Latour, le premier à avoir réalisé une étude de laboratoire (Latour, Woolgar, 1979) rapporte des discussions entre chercheurs autour de la signification d'une pointe dans une distribution : est-elle assez nette pour représenter le phénomène attendu, ou doit-on ne la considérer que comme du bruit ? Dans la discussion interviennent non seulement des critères scientifiques, mais surtout des considérations sociales : le type de revue où on pourra publier les résultats, les réactions supposées des autres spécialistes du problème, etc. Ces observations sont certainement importantes, mais leur portée est peut-être moindre que ne le prétendent leurs auteurs.

Que certains résultats donnent lieu à discussion et à négociation entre chercheurs, et que leur publication n'en fasse pas état, n'est pas contestable, et c'est certainement un des grands mérites des relativistes d'avoir attiré l'attention sur ce point. Mais ils semblent ignorer les cas, et il en existe certainement, où la négociation n'est pas nécessaire, où une conclusion unique s'impose. Et même si une négociation s'avère nécessaire, son issue est soumise à certaines contraintes. Étant donné un ensemble de résultats, d'observations ou de mesures, certaines conclusions sont possibles, et il est vrai que souvent il n'y en a pas qu'une seule, mais d'autres sont exclues parce qu'incompatibles avec les données. C'est un problème analogue à celui de l'indétermination de la traduction, souvent évoqué dans ce contexte : plusieurs textes de la langue d'arrivée sont compatibles avec le texte de départ, mais on ne peut quand même pas dire n'importe quoi, et il y a des énoncés qui n'acceptent qu'une seule traduction.

Peut-être, sur ce point, les conclusions des relativistes sont-elles marquées par le type de données empiriques sur lesquelles ils s'appuient, qui sont le plus souvent des analyses de controverses. Il est vrai qu'un consensus immédiat (cela se produit aussi) donne peu de prise à l'analyse sociologique. Toutefois, en se centrant presque uniquement sur des controverses, on risque de donner une image exagérément problématique de la science, plus problématique en tout cas que si on partait de données plus diverses. Ce n'est pas parce qu'il y a parfois négociation qu'il y en a toujours, et une négociation ne peut pas avoir n'importe quelle issue. Une manière de mener jusqu'au bout une analyse sociologique sans postuler, explicitement ou non, que les facteurs sociaux sont les seuls en jeu, pourrait consister à admettre que la " réalité ", sans déterminer entièrement 
les conclusions qu'on peut tirer d'une expérience, leur impose des contraintes variables selon les situations, laissant plus ou moins de place à l'influence des facteurs sociaux. Mais, pour s'en rendre compte, il faudrait entrer dans le détail du contenu des arguments, et non se contenter de constater leur existence ${ }^{17}$.

À côté de recherches sociologiques plus classiques qui cherchent à mettre en évidence l'influence de la société globale sur les croyances scientifiques, l'existence de ces recherches micro-sociologiques, qui ne tiennent compte que du contexte immédiat constitué par la communauté scientifique, transforme complètement le problème des déterminations internes ou externes, vieux problème de l'histoire des sciences. Classiquement, on identifie d'une part facteurs externes et facteurs extra-scientifiques, et d'autre part facteurs internes et rationalité, nécessité logique ou, plus largement cognitive. Or, les analyses que nous venons de mentionner, et c'est là un de leurs intérêts, brouillent cette distinction. Lorsque Pickering, sur lequel nous reviendrons plus loin, accorde un rôle explicatif central au désir de développer le paradigme avec lequel on travaille, est-ce vraiment du « social » en tant qu'opposé à du « cognitif »? Les négociations sur la réplication qu'analyse Collins font appel, quoi qu'il en dise, à des arguments de type logique. Certes, une communauté scientifique, comme toute communauté, impose des normes à ses membres. En ce sens, le comportement d'un chercheur qui se soumet à ces normes pour être reconnu de ses collègues est bien déterminé par le contexte social. Mais ces normes sont, entre autres, cognitives : elles exigent par exemple de se conformer à des critères de cohérence, de recourir à une certaine logique. Lorsqu'un chercheur s'efforce de présenter une argumentation rigoureuse pour justifier ses conclusions et les faire accepter par la communauté, obéit-il à une nécessité logique ou à des exigences sociales? Les deux coüncident, et c'est peut-être une des spécificités de la communauté scientifique de faire de la rationalité (ou d'une forme de rationalité) une norme sociale ${ }^{18}$.

17. Savoir jusqu'à quel point un sociologue de la science doit connaître et comprendre la discipline qu'il étudie est matière à discussion. Certains (par exemple KnorrCetina, 1981) affirment n'en rien savoir. Ce serait pour eux une protection contre la tentation de partager le point de vue des chercheurs qu'ils étudient au lieu de prendre la distance nécessaire pour l'étudier et l'expliquer. Mais ces chercheurs, eux, travaillent sur des contenus, et en l'ignorant on se ferme a priori à un certain type d'explication.

18. On s'aperçoit du caractère social de ces normes lorsque, de l'aveu même des chercheurs qui s'y conforment, on continue à les appliquer jusque dans des cas où elles ne seraient pas " logiquement" justifiées, comme par exemple lorsque des psychologues utilisent des tests statistiques qui ne correspondent pas exactement à leurs besoins mais qui sont nécessaires pour se faire publier dans certaines revues. Le besoin de disposer de normes de jugement claires, explicites, valables pour tous, est nécessaire au bon fonctionnement de la communauté, et conduit à faire d'une technique particulière une règle générale. 


\section{VII. - ACTIVITÉ SCIENTIFIQUE ET INTÉRÊTS}

Montrer que le contexte social détermine le contenu des croyances scientifiques se fait souvent par analogie, en mettant en évidence, comme le fait Forman, des similitudes entre ces croyances et d'autres qu'on rencontre dans des cercles plus larges. On peut aussi chercher à prouver que ces croyances sont utiles, fonctionnelles, soit pour la société tout entière, soit, comme le font certains marxistes, pour une catégorie sociale particulière. Mais on peut aussi ne pas se contenter de ce degré de globalité. Les sociologues de la science actuels se rattachent presque tous aux courants ou aux théories sociologiques qui cherchent à se placer « du point de vue de l'acteur ». Ils vont donc s'efforcer de montrer comment les scientifiques eux-mêmes, dans leur activité, réalisent ce lien entre le contexte social et les connaissances qu'ils produisent.

L'effort le plus systématique pour atteindre cet objectif recourt à la notion d'intérêt. L'origine de l'utilisation actuelle de ce terme dans le cadre de la sociologie de la connaissance revient à Habermas $(1968,1973)$ qui a introduit la notion d'" intérêts producteurs de connaissance " dont il distingue trois catégories : les intérêts pour la prévision et la domination, qui orientent les sciences de la nature, les intérêts pour la légitimation, à l'œuvre dans ce qu'il appelle les " sciences herméneutiques ", c'est-à-dire l'histoire et certaines sciences sociales, et les intérêts pour la libération, qui devraient donner des " sciences critiques" en particulier une sociologie dont il souhaite le développement. Ces distinctions lui servent en particulier à la critique de ce qu'il appelle " positivisme » dans les sciences sociales, et qui consiste à vouloir appliquer à l'étude de l'homme ou de la société les méthodes des sciences de la nature. Comme celles-ci sont guidées par les intérêts pour la domination, justifiés quand il s'agit de maîtriser la matière, elles ne peuvent donner, appliquées à l'homme, que des techniques manipulatrices, et ne serviront qu'à le dominer, en le traitant comme l'objet d'une causalité mécanique et pas comme un sujet libre.

On voit que cette perspective, beaucoup plus complexe que ce qu'en disent ces quelques lignes, n'est pas du tout un relativisme. Habermas ne conteste pas la validité des connaissances obtenues par les méthodes "positivistes" mais critique seulement leur finalité et l'importance qu'elles ont prise dans notre société.

Il en va tout autrement des sociologues de la science dont il est question ici, pour qui les intérêts déterminent non seulement les objectifs généraux de la science, mais règlent le détail de l'activité et des choix des chercheurs. Mc Kenzie (1981) utilise cette notion à deux niveaux. En premier lieu, il prend en considération les intérêts cognitifs des chercheurs qui orientent directement leur activité. C'est ainsi qu'il explique comment Bravais n'a pas vu toutes les implications du coefficient de corrélation qu'il avait pourtant " découvert ", alors que Pearson, à la suite de Galton, lui a consacré des travaux importants et en a fait un instrument essentiel de ses recherches en statistique. C'est que Bravais n'était pas statisticien (la statistique mathématique n'existait pas à son époque) mais spécialiste du calcul d'erreurs. Il s'était trouvé confronté à un problème concernant les erreurs à deux dimensions, cas très rare dans son domaine. Ses intérêts cognitifs ne l'incitaient pas à approfondir la question, qui était pour 
lui marginale, et n'ouvrait pas de perspectives nouvelles. Pearson, par contre, s'intéressait aux distributions à deux dimensions et ses « intérêts cognitifs » l'ont poussé à développer tous les moyens possibles de les décrire. Mais cet intérêt cognitif lui-même peut être rattaché à d'autres, des intérêts sociaux. Pearson était eugéniste, il était passionné par les problèmes de l'hérédité et de l'évolution. Les distributions à deux dimensions l'intéressaient tout d'abord parce qu'elles permettaient d'étudier les relations entre générations et de décrire les similitudes entre parents et enfants, essentielles dans sa perspective. Ces préoccupations eugénistes sont ensuite rattachées, par Mc Kenzie, aux intérêts de la classe sociale à laquelle appartenait Pearson, la bourgeoisie cultivée luttant contre l'aristocratie. On voit la chaîne causale qui rattache la situation d'un groupe social à la mise au point d'un concept statistique aussi abstrait que le coefficient de corrélation.

L'analyse de Mc Kenzie est fine et bien documentée, convaincante, mais, comme pour Forman, on peut se demander ce qu'il explique réellement. Veut-il dire que sans la position particulière de la bourgeoisie cultivée anglaise, le coefficient de corrélation n'aurait pas vu le jour ? Étant donné la diversité de ses usages actuels, tout à fait éloignés des préoccupations de Pearson, cela semble peu probable. Il est beaucoup plus raisonnable de penser que d'autres chercheurs, ayant des intérêts sociaux tout à fait différents, auraient, eux aussi, rencontré des distributions à deux dimensions, et que l'un d'eux aurait trouvé le coefficient de corrélation, comme Pearson. Peut-être tout ce qu'explique Mc Kenzie, ce sont les accidents historiques qui se trouvent avoir produit la notion de corrélation et de sa mesure, mais pas une dynamique plus générale dont ça a été la concrétisation.

Pickering (1981, 1984 a, 1984 b), qui travaille sur l'histoire de la physique contemporaine, se réfère, lui aussi, à la notion d'intérêts, mais ceux-ci sont d'une autre nature. Pour lui, les intérêts des chercheurs visent à poursuivre leur activité, ce qui, pour des physiciens, implique à la fois la possibilité de développements théoriques et celle d'expériences nouvelles.

Dans son détail, cette conception des intérêts est solidaire d'une conception du développement scientifique dérivée de celle de Kuhn. Celui-ci, dans la deuxième édition (1970) de sa Structure des révolutions scientifiques, a introduit comme élément essentiel d'un paradigme la notion d'《 exemple " (exemplar). Il s'agit d'une solution-type, reconnue par la communauté, à une catégorie de problèmes qui se posent à elle. Les nouveaux problèmes sont résolus par analogie avec l'exemple, dont le champ d'application va ainsi s'étendre. Dans certains cas, l'exemple peut être une théorie ou un modèle, ou des éléments de méthode, dans d'autres cas, ce pourra être moins élaboré et se limiter à quelques idées directrices qui orientent la recherche de solutions. Ces « exemples " sont en général complexes, donc multidimensionnels, ce qui permet à des sous-communautés qui travaillent au développement d'exemples différents d'avoir des points en commun. La science, dans cette perspective, se développe à la fois en étendant, par analogie, le champ d'application d'un exemple, et en établissant des liens entre des exemples différents.

Pour Pickering, l'intérêt des chercheurs est donc à la fois de se poser de nouvelles questions et, en les rattachant aux exemples reconnus par leur 
communauté, de développer ces derniers, ce qui permettra, ensuite, de faire apparaître encore des problèmes nouveaux.

Appliquant cette conception à l'explication de la réaction de la communauté des physiciens des hautes énergies à deux modèles proposés, vers 1974, pour rendre compte de phénomènes nouveaux récemment observés, il écrit, pour expliquer le choix qui s'est finalement opéré :

«Bien que les réactions des tenants de la couleur [un des deux modèles concurrents] fussent logiquement correctes, on peut les qualifier de 'sociologiquement ad hoc' c'est-à-dire qu'elles ne peuvent coïncider avec les intérêts existants... car elles ne réalisèrent aucune connexion analogique avec des ensembles de pratiques existantes, et ne réussirent à créer aucun ensemble nouveau de pratiques » (Pickering, 1981, trad. frçse in Pandore, 1985, p. 92).

Si on s'en tient là, il n'y a rien dans la conception de Pickering que refuserait le plus strict des rationalistes. Quoi de plus normal, du point de vue de ceux-ci, que d'être guidé par le souci d'étendre le champ d'application de sa théorie ou de son modèle ? Cet intérêt est bien interne à l'activité scientifique, et on ne conçoit guère de recherche où il serait absent. Mais Pickering va plus loin, au moins sur deux points. Premièrement, il prend en considération un fait pratiquement ignoré par les épistémologistes, les anticipations des chercheurs. L'intérêt des chercheurs, pour lui, n'est pas seulement, comme chez Lakatos, par exemple, d'expliquer une plus grande part du connu mais, puisqu'il s'agit de pouvoir poursuivre leur activité, de choisir la voie qui a des chances d'être la plus féconde, ce qui introduit une dimension temporelle en général négligée. Et c'est là que le relativisme de Pickering apparaît : on va définir comme " réalité » ce qui permettra le plus d'anticipation, ce qui assure à la science la poursuite de l'activité. Comme il le dit à propos de la mise en évidence des courants neutres en 1973, «... particle physicists accepted the existence of the neutral current because they could see how to ply their trade more profitably in a world in which the neutral current was real " $(1981$, p. 87). La " réalité " des courants neutres est donc déterminée par les intérêts des chercheurs. Mais cela ne suffit pas, évidemment ; il faut encore préciser comment les physiciens ont réussi à rendre les courants neutres "réels ". C'est là le deuxième apport original de Pickering: par une discussion très technique, il s'efforce de montrer que la conclusion relative à l'existence des courants neutres ne s'imposait pas strictement à partir des expériences réalisées, qu'il a fallu affaiblir certains critères, et que, en fait, c'est la croyance dans la réalité des courants neutres qui a déterminé les critères retenus ${ }^{19}$.

19. Cette conclusion a été contestée, sur le plan technique, par certains physiciens qui soutiennent que l'affaiblissement des critères était justifié par l'accroissement de taille des appareils utilisés, où les bruits de fond étaient atténués. Nous n'avons évidemment pas la possibilité de trancher sur ce point. Remarquons seulement que, dans cet exemple, le sociologue, malgré sa volonté d'extériorité, ne peut éviter de prendre position sur des points techniques, internes à la discipline qu'il étudie. 
Nous nous sommes étendu sur le cas de Pickering pour plusieurs raisons. La première est qu'il est un des rares sociologues à recourir à un facteur explicatif qui soit propre à l'activité scientifique. Nous avons vu ci-dessus que, à force de ne voir dans la recherche que des stratégies, des négociations, le champ d'action de facteurs externes, la plupart des sociologues sont incapables d'en montrer la spécificité. Or, les intérêts, tels que les conçoit Pickering, sont bien propres aux chercheurs. Certes, presque tous les groupes organisés, et entre autres les groupes professionnels, visent à se perpétuer, objectif qui vient s'ajouter aux objectifs manifestes et parfois peut se trouver en contradiction avec eux. Mais les intérêts de Pickering visent à perpétuer une activité particulière, celle des chercheurs dont l'objectif manifeste est de produire des connaissances, ce qu'il interprète comme l'extension des exemples. On saisit là quelque chose qui est plus spécifique, plus propre aux scientifiques, que les autres explications ou descriptions proposées.

Le rapprochement de la conception des intérêts chez Mc Kenzie et chez Pickering met en évidence des divergences importantes, qui dépassent ces deux auteurs. Nous avons déjà été amené à distinguer l'explication par le contexte large de la société globale, ou par le contexte plus immédiat de la communauté scientifique. Mc Kenzie recourt au premier type d'explication, Pickering au second. On peut faire l'hypothèse qu'il ne s'agit là ni d'une différence dans les orientations individuelles, ni dans les théories différentes, mais dans les objets de leurs recherches. Mc Kenzie étudie l'émergence d'une nouvelle discipline, la statistique mathématique. Forman, l'autre exemple " externaliste " que nous avons présenté, traite d'un bouleversement important de la physique. Latour, lorsqu'il mêle les facteurs scientifiques, sociaux, politiques, pour rendre compte du pasteurisme, étudie une révolution profonde dans la médecine. À l'opposé, Pickering étudie la physique des hautes énergies à une période où, certes, elle se transforme profondément, mais sans remettre en cause ses fondements. Collins, étudiant les expériences sur la détection des ondes gravitationnelles, Latour lorsqu'il décrit l'activité du laboratoire de neurochimie qu'il a observé, ont eux aussi pour objet ce qu'on peut appeler, après Kuhn, la " science normale ». Cela suggère que les changements importants, qu'il s'agisse d'innovations ou de révolutions, relèveraient d'explications où les facteurs externes jouent un rôle important, alors que le développement « normal » dans des domaines bien constitués dépendrait plus d'une dynamique essentiellement interne.

Cette hypothèse permet de considérer dans une perspective nouvelle la controverse, classique en histoire des sciences, entre "externalistes" et " internalistes ". Si elle était confirmée, il ne s'agirait pas de deux positions mutuellement exclusives, mais d'explications complémentaires dont le poids varie selon les problèmes et les circonstances. La double opposition facteurs externes, sociaux/facteurs internes, cognitifs, devient alors beaucoup moins claire. Où placer les processus internes à la communauté scientifique ? La distinction est d'autant plus difficile à établir que des normes sociales, imposées par un groupe, peuvent être de nature cognitive. La communauté scientifique, prise dans son ensemble, impose à ses membres, sous peine de non-reconnaissance, de se conformer à certaines règles de méthode et d'avoir le souci de la preuve. Cette exigence 
est-elle sociale ou cognitive ? Les deux, certainement, et selon les cas, un aspect peut prévaloir sur l'autre, comme par exemple lorsque la communauté, par l'intermédiaire des normes de publication, par exemple, impose certaines procédures au-delà des cas où les seules exigences logiques les imposeraient.

On voit que l'explication par les intérêts permet de poser des problèmes de très grande portée, et pour cela elle est certainement utile et féconde. Mais elle se heurte à un obstacle extrêmement important : la difficulté qu'on rencontre à montrer quels sont les intérêts en jeu dans une situation donnée. C'est une difficulté que les sociologues connaissent bien, en termes plus généraux, à propos de l'explication des actions par les intentions, notion très voisine. D'une part, cette notion semble devoir faire partie de tout système explicatif réaliste, qui prend en considération des sujets actifs, et pas simplement les objets d'une causalité abstraite. D'autre part, établir quelles sont les intentions, ou quels sont les intérêts, d'un acteur à un moment donné, se heurte à des obstacles empiriques presque insurmontables. Le plus souvent, et c'est le cas des auteurs mentionnés ici, les intérêts sont déterminés à partir des actions, et de leur vraisemblance dans le contexte étudié. Mais on voit ce que cela a de circulaire : si on a besoin de connaître les actions pour en inférer les intérêts, expliquer celles-là par ceux-ci devient tautologique. Sortir de ce cercle en interrogeant les acteurs n'aiderait guère : les intérêts en question sont de ceux dont on n'est guère conscient. Dans certains cas, particulièrement dans la conception de Pickering, cette explicitation par les acteurs eux-mêmes serait impossible : cela impliquerait qu'ils acceptent que la réalité sur laquelle ils travaillent, et qui justifie leur activité, est en fait construite par eux. Cela irait précisément à l'encontre des intérêts que l'on veut mettre en évidence. Comme tous les sociologues " compréhensifs ", les relativistes s'appuient, en définitive, sur la vraisemblance de leurs analyses et de leurs conclusions, sur leur capacité à fournir, des épisodes qu'ils étudient, des exposés clairs, intelligibles et acceptables.

\section{VIII. - INFLUENCE DU CONTEXTE ET DÉVELOPPEMENT Ȧ LONG TERME DE LA SCIENCE}

Tous les travaux empiriques des relativistes, qu'ils soient sociologiques ou historiques, tendent à montrer la dépendance de l'activité scientifique par rapport au contexte social, quelle que soit la manière dont ce contexte est défini. $\mathrm{Si}$ on prend cette détermination au sérieux, cela veut dire que dans d'autres circonstances d'autres connaissances auraient été produites et acceptées comme valides. C'est bien cela qui justifie l'emploi du terme " relativisme " : plusieurs discours sont possibles, qui seront chacun jugés vrais ou faux selon les conditions, sans qu'on puisse se référer à quoi que ce soit qui transcende ces conditions et permette un jugement absolu.

Comme toute affirmation portant sur l'histoire, celle-ci n'est ni confirmable ni réfutable. On ne voit pas à quelles autres conditions comparer celles qui se sont produites, et où est apparue la science que nous connaissons. Mais si 
on admet la détermination par le contexte, il reste à se demander comment la connaissance scientifique se maintient alors que les conditions changent. La situation de la bourgeoisie cultivée anglaise n'est plus la même qu'à la fin du XIX' siècle, et pourtant on continue à utiliser les instruments statistiques élaborés par Galton et Pearson et même à les développer. L'idéologie antiscientifique de l'époque de Weimar dont Forman montre l'influence, a régressé, s'est transformée, a resurgi sous d'autres formes, a touché selon les époques des fractions plus ou moins importantes de la société occidentale et de la communauté scientifique, et néanmoins on continue à accepter la mécanique quantique, à travailler avec elle et à la développer. Le succès de Pasteur est peut-être attribuable au fait qu'il apportait une réponse aux problèmes des hygiénistes, mais maintenant, ceux-ci ont disparu et pourtant la médecine est restée pasteurienne. Il y a là un problème fondamental dans la perspective relativiste : comment des croyances produites, construites, dans des conditions particulières leur survivent-elles ? Et elles ne survivent pas passivement, simplement comme une foi qui se transmettrait sans changement ; la science se développe, de façon qu'il faut bien admettre comme étant cumulative en grande partie. Les connaissances élaborées à un certain moment servent à la construction de celles qui s'y ajoutent ou même de celles qui les remplacent. Certes, certaines connaissances sont rejetées; ce sont celles dont on voit après coup trop facilement le lien avec le contexte social. C'est le cas, par exemple, des mesures de capacités crâniennes effectuées par Morton au milieu du XIX siècle, trop manifestement biaisées par ses présupposés sur l'infériorité des Noirs. Il n'en reste plus rien.

Ce serait maintenant une tâche importante pour la sociologie, et peut-être plus encore pour l'histoire des sciences, de comprendre comment certaines conclusions sont rejetées alors que d'autres, élaborées dans le même contexte, se maintiennent et se révèlent fécondes. Peut-être verrait-on alors que le social n'explique pas tout. Mais cela implique un mode d'approche qui ne soit pas exclusivement sociologique : si on ne cherche qu'une causalité sociale, ce n'est qu'elle qu'on trouvera.

Benjamin MATALON, Université de Paris VIII, Groupe d'études et de recherches sur la science (E.H.E.S.S., associé au C.N.R.S.). 


\section{BIBLIOGRAPHIE}

Bachelard (Gaston), La Formation de l'esprit scientifique, Paris, Vrin, 1934.

BARnes (Barry), Scientific Knowledge and Sociological Theory, London/Boston, Routledge \& Kegan Paul, 1974.

BARNES (Barry), Interests and the Growth of Knowledge, London/Boston, Routledge \& Kegan Paul, 1977.

BARNES (Barry), T. S. Kuhn and Social Science, New York, Columbia University Press, 1982.

BARNES (Barry), Bloor (David), "Relativism, Rationalism and the Sociology of Knowledge ", in Martin HOLLIS, Steven LUKES, eds, Rationality and Relativism, Oxford, Basil Blackwell, 1982.

BEN DAvid (Joseph), Collins (Randall), "Social Factors in the Origins of a New Science : the Case of Psychology ", American Sociological Review, XXXI, 1966, p. 451-465.

BLoOR (David), Knowledge and Social Imagery, London, Routledge \& Kegan Paul, 1976.

BloOR (David), "Sociologie de la logique ou les limites de l'épistémologie ", Pandore, 2, 1983 (trad. du précédent).

BORKENAU (Franz), Der Übergang vom feudalen zum bürgerlichen Weltbild. Studien zur Geschichte der Philosophie der Manufakturperiode, Paris, F. Alcan, 1934 (« Schriften des Instituts für Sozialforschung ").

Brannigan (Augustine), The Social Basis of Scientific Discoveries, Cambridge, Cambridge University Press, 1981.

BruSH (Stephen G.), The Temperature of History, New York, Burt Franklin, 1978.

Callon (Michel), "Struggles and Negotiations to Define what Is Problematic and what Is not : the Socio-Logic of Translation ", in Karin KNORR, Roger KROHN, Richard Whitley, eds, The Social Process of Scientific Investigation, Sociology of the Sciences, Yearbook, IV, 1980, Dordrecht, D. Reidel, 1981, p. 197-219.

Cole (Stephen), Cole (Jonathan R.), Social Stratification in Science, Chicago, University of Chicago Press, 1973.

Collins (Harry M.), "The Seven Sexes : a Study in the Sociology of Phenomenon, or the Replication of Experiment in Physics ", Sociology, 9, 1975, p. 205-224.

Collins (Harry M.), "Stages in the Empirical Programme of Relativism ", Social Studies of Science, $11, n^{\circ} 1,1981$ (a), p. 3-10.

Collins (Harry M.), "Son of Seven Sexes : the Social Destruction of a Physical Phenomenon ", Social Studies of Science, $11, \mathrm{n}^{\circ}$ 1, 1981 (b), p. 33-62.

Collins (Harry M.), Changing Order. Replication and Induction in Scientific Practice, London/Beverly Hills/ New Delhi, Sage Publications, 1985.

Farley (John F.), Geison (Gerald R.), "Science, Politics and the Spontaneous Generation in Nineteenth Century France : the Pasteur-Pouchet Debate ", Bull. of the History of Medicine, 48, 1974, p. 161-198 (trad. frçse, PANDORE, 1982).

Feyerabend (Paul), "Consolations for the Specialist", in Imre LaKatos, Alan Musgrave, eds, Criticism and the Growth of Knowledge, Cambridge, Cambridge University Press, 1970, p. 197-230.

Feyerabend (Paul), Against Method: Outline of an Anarchistic Theory of Knowledge, Londres, New Left Books, 1975 (trad. frçse, Paris, Le Seuil, 1979).

Feyerabend (Paul), Science in a Free Society, London, New Left Books, 1978.

FORMAN (Paul), "Weimar Culture, Causality, and Quantum Theory, 1918-1927 ", Historical Studies in the Physical Sciences, 3, 1971, p. 1-115.

Habermas (Jürgen), La Technique et la science comme idéologie, Paris, Gallimard, 1973

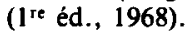


Habermas (Jürgen), Knowledge and Human Interests, London, Heinemann, 1972 (trad. frçse, Paris, Gallimard, 1973).

Hagstrom (Warren O.), The Scientific Community, New York, Basic Books, 1965.

Hendry (John), "Weimar Culture and Quantum Causality ", History of Science, 18, part $3, \mathrm{n}^{\circ} 41,1980$, p. 155-180.

HESSEN (Boris), "The Social and Economics Roots of Newton's "Principia " ", in Nicholas I. Bukharin, et al., Science at the Cross-Roads, 1931, Londres, Frank Cass, $2^{d}$ ed. 1971.

Hollis (Martin), "The Social Destruction of Reality ", in M. HoLlis, S. LuKES, eds, Rationality and Relativism, Oxford, Basil Blackwell, 1982, p. 67-86.

Hollis (Martin), LUKES (Steven), eds, Rationality and Relativism, Oxford, Basil Blackwell, 1982.

KOYRÉ (Alexandre), Études d'histoire de la pensée scientifique, Paris, Gallimard, 1973.

KNORR-CETINA (Karin D.), The Manufacture of Knowledge. An Essay on the Constructivist and Contextual Nature of Science, Oxford, Pergamon Press, 1981.

KUHN (Thomas S.), The Structure of Scientific Revolutions, Chicago, University of Chicago Press, 1962, $2^{d}$ ed. 1970 (trad. frçse, Paris, Flammarion, 1983).

Lakatos (Imre), The Methodology of Scientific Research Programmes, Cambridge, Cambridge University Press, 1978, I.

LAKATOS (Imre), "Falsification and the Methodology of Scientific Research Programmes ", 1970, in 1. LAKATOS, op. cit. supra, p. 8-93.

LAKatos (Imre), "History of Science and its Rational Reconstruction ", 1971, in 1. LAKATOS, op. cit. supra, p. 102-138.

Latour (Bruno), "Comment redistribuer le Grand Partage ? ", Revue de synthèse, CIV, 110 , avril-juin 1983, p. 203-236.

LATOUR (Bruno), Les Microbes : guerre et paix; suivi de Irréductions, Paris, A. M. Métailié, 1984 (“Pandore ").

Latour (Bruno), Woolgar (Steve), Laboratory Life: the Social Construction of Scientific Facts, London/Beverly Hills, Sage Publications, 1979.

LaUdan (Larry), Progress and its Problems : towards a Theory of Scientific Growth, London, Routledge \& Kegan Paul, 1977.

LÉCUYER (Bernard-Pierre), "Bilan et perspectives de la sociologie de la science dans les pays occidentaux ", Archives européennes de sociologie, 19, $\mathrm{n}^{\circ} 2,1978$, p. 257-336.

LEMAine (Gérard), Darmon (Gérard), El Nemer (Saba), Noopolis. Les laboratoires de recherche fondamentale : de l'atelier à l'usine, Paris, C.N.R.S., 1983.

LUKACs (Georg), Geschichte und Klassbewusstsein, 1924, trad. frçse, Histoire et conscience de classe, Paris, Minuit, 1960.

Lukes (Steven), "On the Social Determination of Truth ", in Robin HoRTON, Ruth FINNEGAN, eds, Modes of Thought, London, Faber \& Faber, 1973, p. 230-248.

MC KENZIE (Donald), Statistics in Britain, 1865-1930. The Social Construction of Scientific Knowledge, Edinburgh, Edinburgh University Press, 1981.

MASLow (Abraham), The Psychology of Science, Chicago, Gateway, 1969.

MERTON (Robert K.), "Science and Technology in a Democratic Order ", Journal of Legal and Political Science, I, 1942, p. 115-126; repr. sous le titre The Normative Structure of Science, in R. MERTON, The Sociology of Science, Chicago, University of Chicago Press, 1973.

PANDORE (al.), La Science telle qu'elle se fait, Paris, 1982.

Pandore (al.), Les Scientifiques et leurs alliés, Paris, 1985.

Pelz (Donald C.), Andrews (Frank M.), Scientists in Organizations, New York, John Wiley and Sons, 1966.

PICKERING (Andrew), "The Role of Interests in High-Energy Physics: the Choice between Charm and Colour ", in Karin D. KNORR, Roger KROHN, Robert WHITLEY, eds, The Social Process of Scientific Investigation, Sociology of the Sciences, Yearbook, IV, 1980, Dordrecht, D. Reidel, 1981.

PICKERING (Andrew), Constructing Quarks. A Sociological History of Particle Physics, Edinburgh, University of Edinburgh Press, 1984 (a). 
Pickering (Andrew), "Against Putting the Phenomena First : the Discovery of the Week Neutral Current ", Stud. Hist. Phil. Sci., 15, 1984 (b), p. 85-117.

PINCH (Trevor J.), "The Sun-Set : the Presentation of Certainty in Scientific Life ", Social Studies of Science, $11, \mathrm{n}^{\circ} 1,1981, \mathrm{p} .131-158$.

PrICE (Derek DE Solla), Little Science, Big Science, New York, Columbia University Press, 1963.

Salomon (Jean-Jacques), Science et Politique, Paris, Le Seuil, 1970.

STORER (Norman), The Social System of Science, New York, Rinehart and Winston, 1966. Travis (David), "Replicating Replication? Aspects of the Social Construction of Learning in Planarian Worms ", Social Studies of Science, $11, \mathrm{n}^{\circ} 1,1981, \mathrm{p} .11-32$.

WILSON (Bryan), ed., Rationality : Key Concepts in the Social Sciences, Oxford, Basil Blackwell, 1970. 